\title{
Bioética y epilepsia
}

\section{Bioethics and epilepsy}

\author{
Miguel Ángel Collado-Corona, ${ }^{*, \neq}$ Adalberto González-Astiazarán, ${ }^{*}$ Enrique de Font-Réaulx, ${ }^{*}$ \\ Paul Shkurovich-Bialik, ${ }^{*}$ Javier Terrazo-Lluch, ${ }^{*}$ Ernesto Ramírez-Navarrete, ${ }^{*}$ Emilio Arch-Tirado*
}

Citar como: Collado-Corona MÁ, González-Astiazarán A, de Font-Réaulx E, Shkurovich-Bialik P, Terrazo-Lluch J, Ramírez-Navarrete E et al. Bioética y epilepsia. An Med (Mex). 2020; 65 (3): 246-249. https://dx.doi.org/10.35366/95683

\section{RESUMEN}

La importancia que tiene el manejo de los pacientes con epilepsia obliga a conocer cada uno de los principios de la bioética. El objetivo principal es analizar la enfermedad a la luz de estos cuatro principios: beneficencia, no maleficencia, autonomía y justicia, y establecerlos como parte fundamental en el manejo del paciente epiléptico y, particularmente, de aquellos que sufren epilepsia refractaria y que pudiesen ser sometidos a cirugía de epilepsia, así como enfatizar en la importancia de su conocimiento, lo que permitirá una atención de mayor calidad y que esté a favor de estos pacientes.
\end{abstract}

Palabras clave: Epilepsia, cirugía de epilepsia, bioética, principios bioéticos.

Nivel de evidencia: III

\section{INTRODUCCIÓN}

La Liga Internacional contra la Epilepsia la define como la «crisis o ataques recurrentes producidos por una descarga neuronal excesiva, súbita y desordena-

\footnotetext{
* Clínica de Epilepsia del Centro Neurológico.

‡ Miembro del Comité de Bioética. Comisión Nacional de Bioética.

Centro Médico ABC. México.

Recibido para publicación: 30/05/2020. Aceptado: 10/08/2020.

Correspondencia:

Miguel Ángel Collado-Corona

Av. Carlos Fernández Graef Núm. 154, Santa Fe, Contadero, Cuajimalpa de Morelos, 05330, Ciudad de México, CDMX. Teléfono: 5511031600.

E-mail: mcollado@prodigy.net.mx
}

\begin{abstract}
The importance of treatment in patients with epilepsy, obligates learning every principle in bioethics. The main objective is to analyze the disease within this four principles: beneficence, non-maleficence, autonomy and justice, and establish them as a fundamental part on the management of patients with epilepsy and in particularly in those who suffer refractory epilepsy and could be submitted to surgical treatment and emphasize the importance of its knowledge, thus providing an increased quality of attention and better understanding.
\end{abstract}

Keywords: Epilepsy, epilepsy surgery, bioethics, bioethics principles.

Level of evidence: III

da de un grupo de neuronas no relacionadas con enfermedades agudas». ${ }^{2}$

La epilepsia es uno de los trastornos neurológicos crónicos más frecuentes, pues afecta a más de 60 millones de personas en el mundo. Corresponde a una de las principales causas de atención neurológica, situándose inmediatamente después de las demencias neurodegenerativas, la enfermedad vascular cerebral y la migraña. En nuestro país, estudios epidemiológicos han encontrado tasas de prevalencia e incidencia similares a las de otros países en vías de desarrollo, con un número total de sujetos que oscila entre 1.5 y 2 millones. La epilepsia corresponde a un desorden que afecta a sujetos de todas las edades y es particularmente prevalente antes de los dos y después de los 60 años de edad. ${ }^{3}$

Recientemente, en las últimas décadas, se ha reconocido a la bioética como una disciplina esencial 
para ayudar tanto a médicos, como a pacientes a resolver nuevos y complejos dilemas que surgen con el permanente desarrollo de la ciencia y la tecnología.

En nuestro país, existe una iniciativa en la Ley General de Salud que establece la necesidad de crear comités hospitalarios de bioética. Adicionalmente, se dispone que tanto estos comités como los de ética en la investigación serán interdisciplinarios y deberán estar integrados por personal médico de distintas especialidades y por personas de las profesiones de psicología, enfermería, trabajo social, sociología, antropología, filosofía o especialistas en bioética, abogados con conocimientos en la materia y representantes del núcleo afectado o de personas usuarias de los servicios de salud, así como también el número convenido de sus miembros, guardando siempre el equilibrio de género; ellos podrán estar adscritos o no a la unidad de salud o establecimiento. ${ }^{4}$ El Centro Médico ABC cuenta con un comité de estas características, el cual fue creado con el aval de la Comisión Nacional de Bioética, órgano desconcentrado con autonomía técnica y operativa, cuya misión es coadyuvar a desarrollar una cultura bioética en México.

Lo anterior obliga a que el personal que atiende a los pacientes epilépticos conozca el manejo ético de estos pacientes, pues frecuentemente son víctimas del rechazo social, lo que exige un mejor conocimiento de la enfermedad y erradicar los prejuicios que existen alrededor de ésta y de quienes la padecen, basados en los principios de: beneficencia, no maleficencia, autonomía y justicia. ${ }^{1,5}$

\section{ANTECEDENTES}

La bioética en salud constituye un campo de conocimiento y una práctica que tiene como antecedente a la ética médica o aplicada a la actividad profesional del médico y, por consecuencia, a la de los restantes profesionales de la salud. La bioética médica constituye un conjunto de tendencias que son expresión de la extensión de los paradigmas socio-médicos y tecnológico-científicos en la esfera sistemática de la ética en salud sobre la base de valores morales.

Desde la Segunda Guerra Mundial, el complejo proceso de toma de decisiones éticas en medicina ha tenido una creciente institucionalización y ha estado sujeta a regulaciones legales sumamente estrictas y formales. Las atrocidades cometidas en nombre de la ciencia médica contra las personas prisioneras en los campos de concentración «quienes fueron utilizadas como sujetos de experimentación» generaron profundos cuestionamientos éticos, los cuales fueron aliviados parcialmente por el Código de Núremberg, el cual fija primariamente los principios básicos para la investigación con seres humanos que garanticen su protección y, además, establece entre otros, que la participación del sujeto debe ser totalmente voluntaria, al otorgar su consentimiento ante testigos legales con la libertad de abandonar la investigación o los procedimientos terapéuticos en el momento en que él lo decida.

Además, la investigación debe ser trascendente, bien elaborada, con estricto rigor científico y con riesgos muy reducidos de daño, invalidez o muerte $\mathrm{y}$, desde luego, llevados a cabo por investigadores y clínicos altamente calificados. Posteriormente estos principios fueron adoptados en 1948 en la Declaración de Ginebra y en 1964 por la Declaración de Helsinki. Aunque tenemos las mejores intenciones y la conciencia de una historia con grandes errores e ignorancia, seguimos propensos a cometer errores y equivocaciones al calcular el efecto de nuestras acciones y al volver a fallar en el futuro.

Por estas razones, se introdujo entonces la noción de riesgo-beneficio para los sujetos y la evaluación realizada por un comité independiente de investigadores a nivel institucional, lo que obligó a la deontología médica a incluir la capacidad del sujeto para la toma de decisiones que estuviesen relacionadas con su salud, lo que constituye la premisa para el surgimiento de la bioética médica. ${ }^{1}$

Objetivo: conocer y analizar la epilepsia desde el punto de vista de la bioética y promover la aplicación de sus principios fundamentales en el manejo integral de la enfermedad.

\section{BENEFICENCIA}

Este principio o concepto, evidentemente, se refiere a la búsqueda del bien o beneficio del paciente, a la protección de sus derechos y a la obligación de ayuda de las decisiones que requieren, a veces, un análisis de costo-beneficio en la toma de decisiones terapéuticas, tratando de evitar tratamientos y medidas ineficaces o inútiles. Todo ello se deriva de que cada paciente es un fin último en sí mismo y nuestra actividad diagnóstico-terapéutica es un instrumento a su servicio. Lo anterior implica siempre procurar el bien de nuestro paciente, concebido como una obligación, pues somos nosotros los médicos, responsables de él mientras lo atendemos, sin olvidar que tenemos que respetar en todo momento sus valores y proyectos de vida.,

Este principio bioético nos obliga a cumplirlo cuando atendemos a los pacientes epilépticos para buscar procurarles siempre el bien, y así evitar que 
sean relegados, además de procurar que sus derechos también sean respetados, ya que las diversas consecuencias que la enfermedad pudiese tener sobre sus funciones cerebrales superiores, como pudieran ser su conducta, memoria, atención y habilidad intelectual de acuerdo con el tipo de crisis que presente, así como la mala respuesta a los fármacos antiepilépticos pudiesen afectar seriamente su calidad de vida.

Debemos procurar además que tenga acceso fácil y rápido a la atención de alta especialidad cuando así lo requiriese y que el cuerpo médico, al tomar decisiones (cambio de medicamentos, pruebas diagnósticas costosas e inclusive opciones quirúrgicas cada vez que el paciente lo requiera) considere principalmente su bienestar.

En general, este principio forma parte del pensamiento médico invariablemente y durante todo el tiempo de su actividad profesional, al fijarse el ideal de servicio a sus semejantes con profundo sentido humano y moral que busca hacer el bien, y así, en esto encuentra su plena satisfacción. ${ }^{7}$

\section{NO MALEFICENCIA}

Este principio, que es ampliamente conocido por el gremio médico desde la época hipocrática, se refiere a la obligación de no hacer daño a los pacientes con la conocida frase primum non nocere y busca no causar daño a la integridad física y/o moral del ser humano. Sin embargo, en la época actual hay una gran cantidad de conocimientos tanto científicos como técnicos, que no están exentos de riesgo por ser invasivos, y eso los hace potencialmente procedimientos diagnósticos y/o quirúrgicos, los cuales pueden causar algún mal, incluso sin pretenderlo, debido a un diagnóstico precipitado y a la falta de preparación especializada en el campo, por lo que este principio de beneficencia se complementa hoy con el de no maleficencia. Estos preceptos sustentan también la relación médico-paciente, médico-colega, médico-sociedad y médico-responsabilidad.

Particularmente en el caso de los pacientes epilépticos debemos ser muy cuidadosos cuando indiquemos estudios diagnósticos, buscando siempre en primer lugar aquéllos que no sean invasivos o sofisticados, a menos que éstos fueren altamente necesarios e indicados en forma colegiada para el diagnóstico y decisión terapéutica, como sucede de manera puntual con el caso de la cirugía de epilepsia. ${ }^{8}$

\section{AUTONOMÍA}

Éste es el más moderno de los principios de la bioética, ya que surge al implicarse la idea de libertad personal, como consecuencia de la facultad de autogobernarse. Es un derecho moral y legal, y es una capacidad propia de todos los seres humanos para pensar, sentir, decir y opinar sobre lo que juzgan como positivo o bueno y es innato al ser humano.

En este principio se basan sobre todo la alianza terapéutica entre el médico y el paciente y el consentimiento en las pruebas diagnósticas y los tratamientos. Forma parte integral de la beneficencia. El paciente debe tener la capacidad de comprender la información que se le proporciona y las consecuencias de su decisión, así como la capacidad para elaborar los razonamientos necesarios para comunicar su decisión al respecto. Debemos tener la certeza de que el paciente está decidiendo para él, según sus valores, que se le ha transmitido información veraz, en lenguaje claro y comprensible de acuerdo con sus necesidades y sin coerción que le dificulte la toma de decisiones. ${ }^{9}$

Sin embargo, las decisiones y acciones autónomas pueden ser restringidas por factores internos, como en el caso de la epilepsia, que es una disfunción del sistema nervioso central, o externos como la ausencia de información acerca de su diagnóstico y/o tratamiento, o bien, por información errónea, equivocada o incluso engañosa.

Las restricciones internas son aplicables a pacientes con epilepsias refractarias y, por tanto, candidatos a cirugía de epilepsia, a causa del daño neurológico que los acompaña, pero también el factor edad limita frecuentemente su capacidad de autonomía, por lo que el ejercicio de este derecho debe ser ejercido por terceras personas; en esos casos se genera otro dilema ético que puede subsanarse con la solicitud del consentimiento informado a los padres, tutores o representantes legales. ${ }^{10}$

Con lo anterior, se prioriza el respeto al derecho de autonomía de los pacientes dentro del respeto general por su integridad biopsicosocial. Actuar con responsabilidad requiere de la aceptación de los valores individuales y al mismo tiempo del deber de educar a las personas y grupos para concientizarlos de las necesidades individuales de estos pacientes, así como buscar el amparo de las leyes sociales y jurídicas para su protección. ${ }^{10}$

\section{JUSTICIA}

Este principio es reconocido como básico en cualquier sociedad, sea cual fuere su contenido concreto, y se fundamenta en la igualdad de todos los seres humanos, ya que las cargas y beneficios que implica la salud y la enfermedad debieran ser distribuidas de mane- 
ra totalmente igualitaria. La justicia funciona como la medida de la distribución equitativa de derechos y obligaciones, ventajas y desventajas y créditos activos y pasivos entre todos los miembros de la sociedad. ${ }^{10}$

Justicia en salud significa otorgar a cada quien, en el momento preciso, lo necesario con independencia de su estatus social y sin reparar de manera prioritaria en los costos.

$\mathrm{Al}$ aplicar y fomentar el conocimiento científico, la práctica médica y las tecnologías conexas, se deberían potenciar al máximo los beneficios directos e indirectos para los pacientes, los participantes en las actividades de investigación y otras personas involucradas, y se deberían reducir, de la misma manera, los posibles efectos nocivos para dichas personas. ${ }^{6}$

Se habrá de respetar la igualdad en dignidad y derechos fundamental en dignidad y derechos de todos los seres humanos, de tal modo que sean tratados con justicia y equidad.

El principio de justicia entendido desde la solidaridad y la filantropía, aplicado a la cirugía de epilepsia, representa la obligación de compartir todo aquello con lo que se cuenta en materia de recursos para la salud: infraestructura hospitalaria, personal médico y paramédico multidisciplinario con una altísima y comprobada preparación en el campo de la epilepsia y, de manera muy importante, los recursos económicos de donadores altruistas, todos compartiendo el mismo interés en la atención de la población vulnerable y que no tiene acceso a atención en estos importantes recursos.

\section{CONCLUSIÓN}

Hemos analizado a la epilepsia desde los principios fundamentales de la bioética con el fin de estable- cer el conocimiento de estos preceptos y aplicarlo en el manejo médico de esta enfermedad en nuestro centro médico; dejamos de manifiesto la importancia que reviste el estudio y el abordaje de esta enfermedad en el diario acontecer médico para que desarrollemos un trabajo de excelencia en nuestros pacientes.

\section{BIBLIOGRAFÍA}

1. Ruiz de Chávez M. Temas selectos de conbioética. México: Gaceta Conbioética; 2016.

2. Engel J Jr, Pedley TA. Introduction: ¿what is epilepsy? In: Engel J Jr, Pedley TA, editors. Epilepsy: a comprehensive textbook. Philadelphia: Lippincott Raven Publishers; 1997. pp. 1-7.

3. Shkurovich BP, Collado CMA. Avances en el manejo de la epilepsia. Rev Neurol Neurocir Psiquiat. 2017; 45 (1): 26-31.

4. Comisión Nacional de Bioética. Su entender, su quehacer. Debate Bioético. 2007;1(2):34.

5. Gabilondo Zubizarreta FJ. Principios de bioética y ética profesional. Disponible en: http://www.secpre.org/ documentos\%20manual\%2019.html

6. Organización de las Naciones Unidas para la Educación la Ciencia y la Cultura. Proyecto de Declaración Universal sobre Bioética y Derechos Humanos. Adopted by acclamation on 19 October 2005 by the 33rd session of the General Conference of UNESCO [sede web]. UNESCO. Disponible en: http://portal.unesco.org/es/ev.php-URL $\mathrm{ID}=31058 \&$ URL_DO $=$ DO_TOPIC\&URL_SECTION $=201$. html

7. Fernández-Vázquez JM. Código de ética médica. Centro Médico American British Cowdray. México, D.F.: Lito-Grapo; 2006. p. 15.

8. Costa Alcaraz AM, Almendro Padilla C. Los principios de la bioética: autonomía [sede web]. Disponible en: http://www. fisterra.com/formacion/bioetica/autonomia.asp

9. Annas GJ. Globalized clinical trials and informed consent. N Engl J Med. 2009;360(20):2050-2053.

10. Ochandategui-Camejo P. La epilepsia a la luz de la bioética. Revista Electrónica de PortalesMedicos.com [publicación periódica en línea]. 2011. 